University of Wollongong

Research Online

Faculty of Engineering and Information

Faculty of Engineering and Information

Sciences - Papers: Part A

Sciences

$1-1-2013$

\title{
Economical data-intensive service provision supported with a modified genetic algorithm
}

Lijuan Wang

University of Wollongong, Iw840@uowmail.edu.au

Jun Shen

University of Wollongong, jshen@uow.edu.au

Follow this and additional works at: https://ro.uow.edu.au/eispapers

Part of the Engineering Commons, and the Science and Technology Studies Commons

Research Online is the open access institutional repository for the University of Wollongong. For further information contact the UOW Library: research-pubs@uow.edu.au 


\title{
Economical data-intensive service provision supported with a modified genetic algorithm
}

\begin{abstract}
The explosion of digital data and the dependence on data-intensive services have been recognized as the most significant characteristics of the decade. Providing efficient mechanisms for optimized dataintensive services will become critical to meet the expected growing demand. In order to create a cost minimizing data-intensive service composition solution, we design two steps and two negotiation processes over the lifetime of a data-intensive service composition. The solution for the first step is presented in this paper. The proposed service selection algorithm is based on a modified genetic algorithm, which some modifications of crossover and mutation operators are adopted in order to escape from local optima. The performance of the algorithm has been tested by simulations.
\end{abstract}

\section{Keywords}

modified, data, genetic, algorithm, intensive, service, provision, supported, economical

\section{Disciplines}

Engineering | Science and Technology Studies

\section{Publication Details}

Wang, L. \& Shen, J. (2013). Economical data-intensive service provision supported with a modified genetic algorithm. IEEE 2nd International Congress on Big Data (pp. 358-365). United States: IEEE. 


\title{
Economical data-intensive service provision supported with a modified genetic algorithm
}

\author{
Lijuan Wang, Jun Shen \\ School of Information Systems and Technology \\ University of Wollongong \\ NSW, Australia \\ Email:1w840@uowmail.edu.au,jshen@uow.edu.au
}

\begin{abstract}
The explosion of digital data and the dependence on data-intensive services have been recognized as the most significant characteristics of the decade. Providing efficient mechanisms for optimized data-intensive services will become critical to meet the expected growing demand. In order to create a cost minimizing data-intensive service composition solution, we design two steps and two negotiation processes over the lifetime of a data-intensive service composition. The solution for the first step is presented in this paper. The proposed service selection algorithm is based on a modified genetic algorithm, which some modifications of crossover and mutation operators are adopted in order to escape from local optima. The performance of the algorithm has been tested by simulations.
\end{abstract}

Keywords-data-intensive service composition, genetic algorithm, quality of service.

\section{INTRODUCTION}

Big data has become a popular term as the rapid development of Internet, cloud computing, mobile and Internet of things. It is used to describe the exponential growth, availability and use of information, both structured and unstructured. The impact of enormous new sources of data extends to many areas of society, far beyond business, industry, government, science, sports, advertising and public health, with no area being untouched. The IT services industry is already abuzz about Big Data and can now make key business decisions by predicting how customers and competitors customers will behave and how their behavior may change. Obtaining deeper insights into such key business decisions is critical for bettertargeted marketing. Furthermore, Big Data helps to achieve a larger share of profit in terms of both market and customers.

Cloud computing has become a viable, mainstream solution for data processing, storage and distribution. It provides unlimited resources on demand. Considering Big Data and the cloud together, we see a practical and economical way to deal with Big Data, which will accelerate the availability and acceptability of analysis of the data. To put Big Data to work, increasing numbers of companies are starting to use the cloud to publish Big Data as a data service. Many data services in the area of Big Data analytics have now become available and they are called data-intensive services. These data-intensive services consume and produce large data sets. Applications based on data-intensive services have become the most challenging type of applications in service-oriented computing.

In this paper, we address the problem of data-intensive service composition. This paper defines the notion of dataintensive services as Web services that make use of very large data sets as inputs. The data come from a variety of sources. It may be expressed in different schemes, formats, and units. We regard the data owners as data providers and data is stored in data centers. When services make use of the data, the service provider needs to pay the access cost and transfer cost. Providing efficient mechanisms for optimized data-intensive services will become critical to meet the expected growing demand. We make a distinction between cost and other QoS attributes because cost is usually related to other quality attributes and it becomes more important in data-intensive service provision. In traditional service composition, executable services and its input/output data are usually in the same site. Thus the cost for data staging can be neglected or the cost is a constant determined before execution, and service selection algorithms need not consider it. However, in data-intensive service composition, providers charge users depending on the user's location and the amount of data transferred. When composing dataintensive services, optimizing the cost of data is a priority, as data play the dominant role in the data-intensive service composition.

In general, data-intensive service composition will be supported cooperatively by service composers, service providers, and data providers. Different providers need a method to regulate and price their resources, and they all want to have a position in the market whilst maximizing their profits. The services are in a highly dynamic environment where the status and the number of services can change without warning. Thus, it is extremely important to be able to perform dynamic optimization at the same time as a composite service is being executed. We have already done pilot studies in applying bioinspired algorithms to tackle service composition problems [7], [8], [10], [11], [13]. [11] was the first effort to address the lower cost data-intensive service composition problem. Based on our earlier outcomes and other studies, we are designing two steps and two negotiation processes over the lifetime of a data-intensive service composition to get a holistic cost minimization data-intensive service composition solution. The solution for the first step is presented in this paper. A modified genetic algorithm is proposed to select service for data-intensive service composition.

The remainder of this paper is organized as follows: Section II introduces the related work. Section III specifies the problem. Section IV investigates how a modified genetic algorithm could be used to solve the data-intensive service composition problem. Section V shows the performance evaluation. Finally, Section VI concludes this paper and proposes future work. 


\section{RELATED WORK}

The research area of QoS-aware service composition has been attracting much attention in recent years. Most servicecomposition processes involve static price-setting models. To obtain a viable business model for composite services, most researchers expect an increase of dynamic service pricesetting models using non-standard pricing mechanisms, such as auctions [1] and negotiations [3], and an increase in the automation of selling and purchasing processes [2].

The authors of [12] made a comprehensive examination of the profit and quality issues in both static and dynamic pricing scenarios. According to the static pricing policies, users pay a fixed price for a satisfactory composite service. From the service composer's point of view, the profit optimization turns into finding a composite service that not only satisfies the user's requirements but also has the lowest cost. Dynamic pricing policies mean that users pay different prices for composite services with different qualities. There should be a balance between the cost and the price of a composite service. Based on the above assumptions, the authors presented a price-oriented heuristic approach for profit optimization with a recursive 'divide and select' process. The paper [4] discussed the issues surrounding the automation of dynamic electronic service composition by presenting negotiation strategies in the context of multiple simultaneous auctions. The paper [5] presented a service composition agent that bought component services by participating in many English auctions and sold composite services by participating in request-for-quotes reverse auctions. The authors of [6] modeled the composition problem as the winner determination problem in a procurement auction with combinatorial bids and volume discounts. They developed efficient polynomial time algorithms for multiple instances of linear workflow and tree workflow of end-to-end composite service, but in the absence of QoS constraints.

Most of the existing studies consider the service composition problem as an optimization problem. Many different optimization algorithms are applied to solve service composition problems. In addition, these studies assume that service providers pre-determine the price of their services, and this means that they do not consider the economical dynamics of the service composition problems. There is a variety of factors affecting whether a business is profitable or not, including market demand, competition, location and so on, but one of the most important elements is how to manage pricing strategies. Different types of services have different strategies to determine the price. In this situation, the price cannot be known in advance but it is possible to give tariffs expressions, for example, a price per duration or a price per data volume. In the following section our problem statement will be specified.

\section{PRoBlem STATEMENT}

\section{A. The lifetime of data-intensive service composition}

The lifetime of our data-intensive service composition is described in Fig. 1. During the lifetime of our data-intensive service composition, the first step is that the service composer selects a set of service candidates while the service providers select data replicas, and the second step is that the service composer negotiates with multiple service providers while the service providers negotiate with their respective data providers at the same time. The service composer selects a set of elementary services in oder to get a satisfactory composite service, according to its user's QoS requirements. If the solution cannot be found, the service composer will negotiate with several service providers to ask them to change their offers.

There are various ways of assisting service providers to adjust the QoS of their services to a certain level and improve their competitiveness. Fig. 2 shows an example. The triangles represent services that were chosen by the service composer. The QoS taken here to be the response time and price. The service $\mathrm{x}$ in Fig. 2 is not chosen for service contracts. Then the service composer gives the information of the current status of the competition to service providers and asks them to decrease the price of service $\mathrm{x}$. The competitiveness of service $\mathrm{x}$ can be improved if it closes to the black dots by improving one of the QoS attribute (provided as service $\mathrm{x} 1$ or service $\mathrm{x} 2$ ) or improving both QoS attributes (provided as service $\mathrm{x} 3$ or

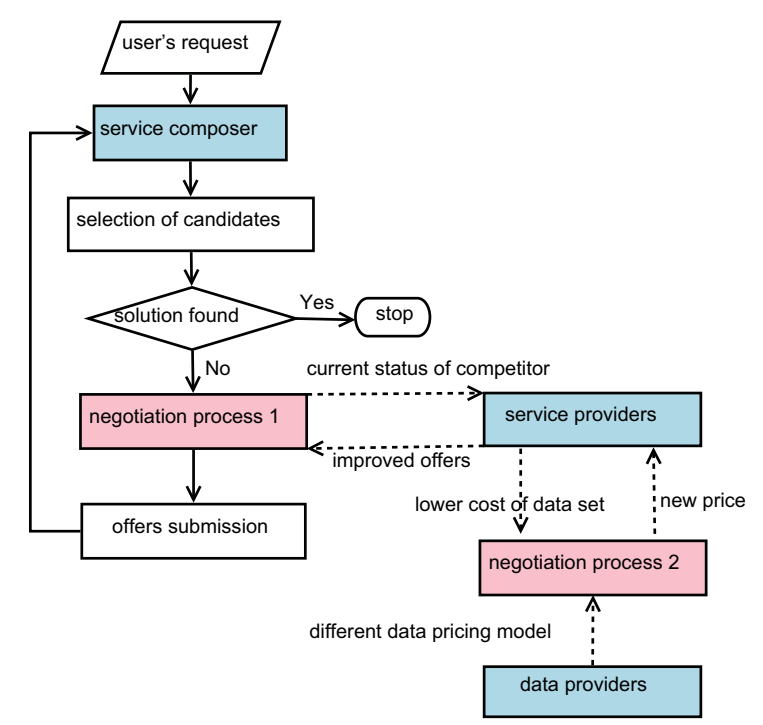

Fig. 1: The lifetime of data-intensive service composition

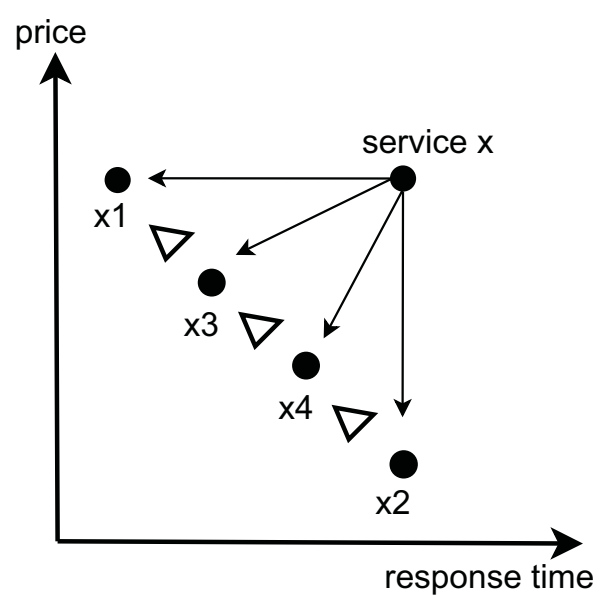

Fig. 2: Various modifications to improve the competitiveness of service $\mathrm{x}$ 
service $\mathrm{x} 4$ ) at the same time. Clearly, these ways typically impose some overhead. For example, using faster servers, or more CPU computation power, or a better network can reduce the response time of services being run on the cloud. Service providers will prefer the strategy that can modify services' QoS levels at minimum cost.

If the service composer negotiates with service providers to ask better offers in the first negotiation process, service providers will negotiate with data providers to ensure obtaining a lower cost for the data sets in the second negotiation process. The data providers use different pricing models to supply data, such as the usage-based pricing model (UB), the package-based pricing model (PB), the flat fee subscriptionbased pricing model (SB), and the combination-based pricing model (CB). The service provider can switch from one pricing model to another back and forth, according to their demands. If service providers choose the flat fee subscription-based pricing model or the combination-based pricing model, they will move the needed data sets closer to their service implementation locations. This can reduce the response time and enhance the usage of bandwidth and other QoS attributes. Normally, it is necessary for the service composition processes to have dynamic pricing models.

\section{B. The model of data-intensive service composition}

The data-intensive service composition problem is modeled as a directed graph, denoted as $G=(V, E, D$, start, end $)$, where $V=\left\{A S_{1}, A S_{2}, \ldots, A S_{n}\right\}$ and $E$ represent the vertices and edges of the graph respectively. There are only two virtual vertices, the start vertex which has no predecessors, and the end vertex which has no successors. Each edge $\left(A S_{i}, A S_{j}\right)$ represent a relationship between $A S_{i}$ and $A S_{j}$, which means that $A S_{i}$ has to finish before invoking $A S_{j}$. Each abstract service $A S_{i}$ has its own service candidate set $c s_{i}=\left\{c s_{i, 1}, c s_{i, 2}, \ldots, c s_{i, m}\right\}, i \in\{1, \ldots, n\}$, which includes all concrete services to execute $A S_{i}$. $D$ represents a set of data sets for services. Fig. 3 gives an example of a directed graph

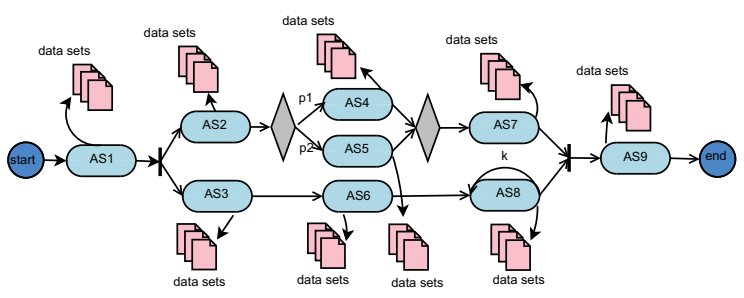

Fig. 3: An example of directed graph for data-intensive service composition

for data-intensive service composition, in which data sets, as the inputs of services, are incorporated. For simplicity, it is assumed that all data sets needed by each service have already been distributed in data centers prior to service composition following a uniform distribution, and we will not deal with the selection of data replicas in this paper. In addition, we will only consider the cost and response time of data-intensive services.

\section{The optimization problem in the two-stage negotiation procedure}

If a feasible solution which satisfies the global constraints does not exist, negotiation is performed in order to determine new quality values for each involved service. In our data-intensive service composition model, there are two-stage negotiation procedure. In the first stage, a service composer negotiates with multiple service providers over each elementary service in a structured one-to-many negotiation process. In the second stage, multiple service providers negotiates with a data provider over each data set in a structured manyto-one negotiation process. In the following, we present the optimization problem of data provider, service provider and service composer in the two-stage negotiation procedure.

1) Data provider: In this paper, we consider the usagebased pricing model, the package-based pricing model, and the flat fee subscription-based pricing model. The payoff function of a data provider is that of maximizing its profit considering the actual income (price paid by data users for each data set) and the actual cost of maintaining the data servers (price paid by the data provider for its data). We consider there are $M$ service providers that offer a data-intensive services $S$, using a set $D T$ of data sets offered by a data provider. The number of data sets charged on the usage-based price is $U B_{m, d t}$, charged on the package-based price is $P B_{m, d t}$, and charged on the flat fee subscription-based price is $S B_{m, d t}$. The data provider optimization problem during a period $T$ is given by (1).

$$
\begin{aligned}
\max \Theta_{d p}^{T} & =\sum_{m \in M} \sum_{d t \in D T}\left(p_{d t} * U B_{m, d t}\right. \\
& \left.+p_{p k} * P B_{m, d t}+f_{d t}^{s t} * S B_{m, d t}\right)-c_{D T}
\end{aligned}
$$

where $c_{D T}$ is the cost of maintaining a set $D T$ of data sets. $p_{d t}, p_{p k}$ and $f_{d t}^{s t}$ are the price of data sets in different pricing models respectively.

2) Service provider: It is assumed that there are $M$ service providers can provide service $S$, the set of which is denoted by $F=\{1,2, \ldots, M\}$. Each provider has two parameters to set as regards $S:\left(p_{i}^{S}, q_{i}^{S}\right) \cdot p_{i}^{S}$ is the price that SP $i$ charges his customers per unit invocation and $q_{i}^{S}$ denotes the QoS attributes (response time, availability or reliability, etc). The price and QoS attributes of service $S$ of all service providers is denoted by the vector $\mathbf{p}=\left\{p_{1}^{S}, p_{2}^{S}, \ldots, p_{M}^{S}\right\}$ and the vector $\mathbf{q}=\left\{q_{1}^{S}, q_{2}^{S}, \ldots, q_{M}^{S}\right\}$ respectively. The demand for service $S$ of SP $i$ depends not only on its own parameters $p_{i}^{S}$ and $q_{i}^{S}$, but also on the prices and QoS offered by its competitors. The demand functions themselves can take on different functional forms. In this work, the demand for services is defined as follows:

Definition 3.1: The demand for a service $S$, denoted as $\varphi_{S}(\mathbf{p}, \mathbf{q}, t)$, is the amount of invocations of $S$ at time $t$.

Naturally, in realistic situations, the demand for a service is measured in time intervals. If a customer invokes service $S$ then $\varphi_{S}(\mathbf{p}, \mathbf{q}, t) \geq 0$, otherwise $\varphi_{S}(\mathbf{p}, \mathbf{q}, t)=0$. The decision of the payment about the required data sets based on the demand of the service: if the demand is low, it is possible to pay the data set for respective access; if the demand is high, then pay flat fee may be more profitable for the service provider. 
The SP $i$ makes profit by charging the service for a price. The profit of service $S$ at time $t$ for SP $i$ is the difference between the revenue and the fee it pays to the data owner and the network owner, which is given by (2).

$$
\theta_{i}^{S}(\mathbf{p}, \mathbf{q}, t)=\varphi_{i}^{S}(\mathbf{p}, \mathbf{q}, t) * p_{i}^{S}(t)-c d_{i}^{S}(t)-c r_{i}^{S}(t)
$$

where $p_{i}^{S}(t)$ is the price of service $S, c d_{i}^{S}(t)$ is the access cost and the transfer cost of all data sets required by service $S$ at time $t, c r_{i}^{S}(t)$ is the service related cost which mainly includes the cost to provision the service $S$ and the cost to process the data sets.

The data sets accessed by services can be paid in different pricing models at any time $t \in[0, T]$ and the total profit of SP $i$ for service $S$ is $\Theta_{i}^{S}(t)=\int_{0}^{T} \theta_{i}^{S}(\mathbf{p}, \mathbf{q}, t) \mathrm{d} t$. The optimization problem of SP $i$ is to maximize the total profit in $[0, T]$ by choosing which pricing model to pay the data sets at any time, which is given by (3).

$$
\max \Theta_{s p}(t)=\int_{0}^{T} \theta_{i}^{S}(\mathbf{p}, \mathbf{q}, t) \mathrm{d} t
$$

It is straightforward that both the demand and the price of a service must be positive numbers. Furthermore, it is necessary to impose an upper and lower bound on the price. As mentioned before, the demand and the price of a service are connected variables: intuitively, as the price for a service increases the demand decreases and vice versa. In order to have a contract with the service composer, the service provider needs to low the price by decreasing the total cost of the service. For service $S$, these bounds can be formulated according to (4) and (5).

$$
\begin{gathered}
\varphi_{S} \geq 0 \\
0 \leq f_{i}\left(q_{i}^{S}\right) \leq p_{i}^{S} \leq p_{\max }^{S}
\end{gathered}
$$

where $p_{\text {min }}^{S}=f_{i}\left(q_{i}^{S}\right)$. The dependence of $p_{\text {min }}^{S}$ on $q_{i}^{S}$ through the function $f_{i}$ will eliminate explicitly policies that will result in negative profits for a service provider. The maximum prices reflect the fact that beyond some reasonable price, the demand will be zero (what ever the price of the service provider and QoS are).

3) Service composer: The service composer needs to select an overall best set of candidate services with sufficient quality at a reasonable price, according to a utility function and QoS constraints. The utility function expresses preferences that prioritize values of the QoS attributes, typically a weighted sum of the normalized QoS attributes. The constraints define requirements regarding the aggregated QoS values of the requested composite service. The service composer can give different optimization goals according to its needs. Then the above problem is turned into a constraint optimization problem. In this research, the objective goal is to find out the feasible selection with the lowest expected cost, and the constraint is the execution time. The optimization problem for the service composer is given by (6).

$$
\max \Theta_{s c}=p_{C S}-\sum_{i=1}^{n} p^{\sum_{j=1}^{m} c s_{i, j} * x_{i, j}}
$$

where $p_{C S}$ is the price of the composite service $C S$, $p^{\sum_{j=1}^{m} c s_{i, j} * x_{i, j}}$ is the price of service $c s_{i, j}$ which is used to implement $A S_{i} . x_{i j}$ is the constraint used to represent only one concrete service is selected to replace each abstract service during the process of service composition, where $x_{i j}$ is set to 1 if $c s_{i, j}$ is selected to replace abstract service $A S_{i}$ and 0 otherwise.

Using a genetic algorithm, the problem of finding a dataintensive service composition solution is considered as an optimization problem, in which the overall fitness value has to be maximized. Formally, the optimization problem is described as follows. Find a solution $C S$ in graph $G$ by replacing each abstract service $A S_{i}$ in $V$ with a concrete service $c s_{i, j} \in c s_{i}$ such that the overall fitness $F(C S)$ is maximized. The fitness function is described in the following section.

\section{DATA-INTENSIVE SERVICE SELECTION BASED ON A MODIFIED GENETIC ALGORITHM}

Genetic algorithms (GAs) belong to the larger class of evolutionary algorithms (EAs), which generate approximate solutions to optimization and search problems by using techniques inspired by the principles of natural evolution: selection, crossover, and mutation. GA is a powerful tool to solve combinatorial optimization problems [9]. It is an iterative procedure based on a constant-size population. In a GA, a population of strings (called chromosomes or the genotype of the genome), which are encoded as candidate solutions (called individuals, creatures, or phenotypes) to an optimization problem, evolves towards better solutions. Each genome is associated with a fitness value based on a fitness function that indicates how close it comes to meeting the overall specification, when compared to other genomes in the population. The fitness value of an individual is also an indication of its chances of survival and reproduction in the next generation. A typical genetic algorithm requires a genetic representation of the solution domain and a fitness function to evaluate the solution domain.

To use a GA to search for a solution to the data-intensive service composition problem, the first step is to encode the problem with a suitable genome. The encoding scheme of chromosomes in this paper is the integer array coding scheme, namely, every chromosome is represented by an integer array with a number of items. Using the integer array coding scheme, any change in the number of service candidates would not influence the length of the genome. Also, this kind of encoding is human-readable and straightforward to represent the service composition solution. Each gene in the chromosome represented an abstract service in the composite service, and the value of the gene represents an assignment of a concrete service for that abstract service. GA works with a set of chromosomes called a population. The whole population moves like one group towards an optimal area, so the GA searches from a population of solutions rather than a single solution.

\section{A. The implementation of a modified genetic algorithm for data-intensive service selection}

The implementation of our genetic algorithm applied to data-intensive service selection performs as follows.

Initialization. Once a suitable representation for the chromosomes has been decided, it is necessary to create an initial population to serve as the first generation. In this paper, 
the initial population is created randomly according to a directed graph. Only one branch of the conditional operations is selected according to a certain probability. When creating the initial population, if one of the branches of the conditional operations is not selected, the values of the genes are set as zero, which indicate the related abstract services are not included in the composite service.

Evaluation. After the initial population is created, every individual in the population is evaluated by a fitness function. Fitness denotes a measure that is used to select individuals for further evolution. Applied to the service selection problem in this paper, fitness is a measure for the utility of the composition solution resulting from the selection of service candidate for each abstract service.

Selection. After the fitness evaluation, a set of individuals are selected which are then used by the crossover operator to produce offspring for the new generation. The selection operator in this paper is the combination of elitism selection and tournament selection. Elitism selection involves copying a few best individuals, unchanged, into the next generation. Tournament selection involves selecting a set of pairs of individuals as parents to breed the remainder of the next generation. We perform two separate tournaments to choose father and mother, respectively. After a set of pairs of parents has been selected, a crossover operation will be executed to produce offspring.

Crossover operation. The crossover operator in this paper is the single point crossover. Suppose there are Npop individuals in the population and the crossover probability is $P C$, then there are $N p o p * P C$ individuals which are replaced by the new offspring, and there are $N p o p *(1-P C)$ individuals which are able to survive to the next generation. The crossover point is created randomly but must be checked as to whether it will create unfeasible solutions. For example, suppose there are twenty service candidates for each abstract service in Fig. 3 and the crossover point is four. The parents and the offspring are given in Fig. 4. The two offspring are unfeasible because all conditional branches are selected in the first offspring, and none of the conditional branches is selected in the second offspring. The checking rule is that there is one and only one branch of each conditional operation to be selected.

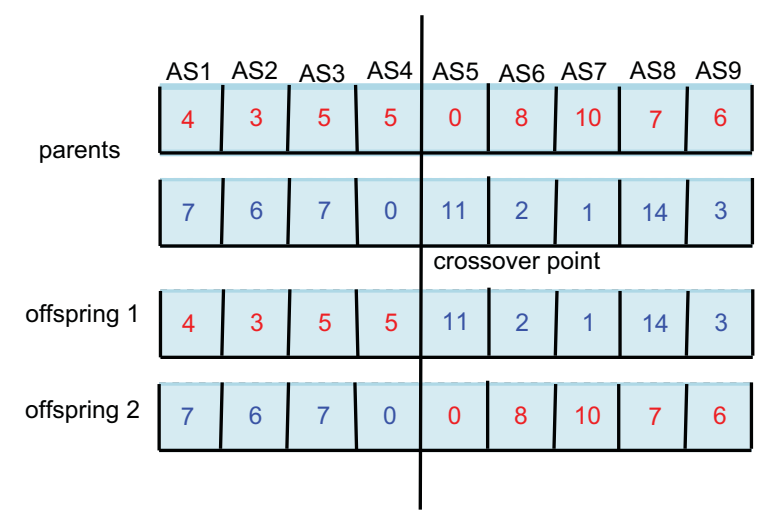

Fig. 4: An example of unfeasible solution generated by crossover operation
Mutation operation. If we only use the crossover operator to produce offspring, unexplored service composition solutions cannot be added into the population. Also, the same value of the gene at the same position of all individuals in the initial population will be kept in future offspring. Thus, it is necessary to adopt a mutation operator. In this paper the mutation policy is proposed as follows. The probability of mutation $(P M)$ is for the locus. The locus for each gene represents its own position in the chromosome. Every locus in each chromosome which was created by the crossover operation is checked for possible mutation by generating a random number between zero and one. If this random number is less than or equal to the given mutation probability, then the value of the gene will be replaced by the assignment of another concrete service in the service candidate set. After the mutation operation, only the chromosome with the greater fitness value will survive between the mother and child chromosome. Before the mutation operation, it is necessary to check whether the value of the gene equals zero. If the value of the gene equals zero, it means the related abstract service is in a conditional branch and it is not selected, so the mutation operator will not be applied to this locus. For example, suppose there are forty service candidates for each abstract service in Fig. 3. The original offspring and mutated offspring are given in Fig. 5. The mutated offspring is feasible when the locus is 7, but it is unfeasible when the locus is 5 . The value of the gene in locus 5 equals zero, which means the abstract service $A S 5$ is in a conditional branch and it is not selected. Thus, the mutation operation cannot be applied to locus 5 of the original offspring.

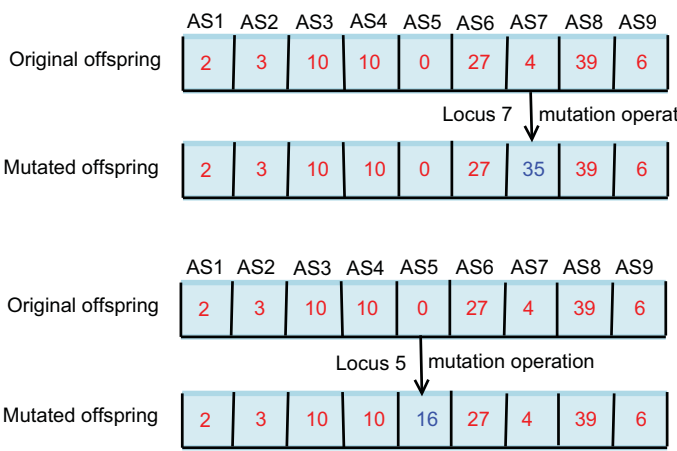

Fig. 5: An example of feasible solution and unfeasible solution generated by mutation operation

Iteration and termination. After the crossover and the mutation operations, the new population is composed of the individuals in the new generation and a few individuals from the previous generation. The fitness of each individual in the new population is evaluated and the whole procedure is repeated until a termination condition has been reached.

\section{B. Fitness function}

The function used to evaluate the quality of a potential solution is called the fitness function. Fitness can be measured in a variety of ways: as a distance, as an error, as a time interval, etc. When using a GA to solve service composition problems, the fitness function always corresponds to QoS 
attributes. As we discussed before, this paper will consider only the cost and response time of data-intensive services. The fitness of a genome $g$ (namely, composite service $C S$ ) is $F(C S)=\sum_{k=1}^{2} \frac{Q_{k}^{M A X}-q_{C S}^{k}}{Q_{k}^{M A X}-Q_{k}^{M I N}} * W_{k}$, where $q_{C S}^{k}$ is the $k$ th aggregated QoS value, computed according to the methods presented in [10]. $W_{k}$ represents weights of $k$-th quality criteria with values provided by the users based on their own preferences, and $\sum_{k=1}^{2} W_{k}=1 . Q_{k}^{M A X}$ and $Q_{k}^{M I N}$ represent the maximal value and the minimal value of the $k$-th QoS attributes of all the possible solutions, respectively. They are computed according to (7).

$$
\begin{aligned}
& Q_{k}^{M I N}=\sum_{i=1}^{n} Q_{k, i}^{\text {min }}=\sum_{i=1}^{n} \min _{\forall c s_{i, j} \in c s_{i}} q_{i j}^{k} \\
& Q_{k}^{M A X}=\sum_{i=1}^{n} Q_{k, i}^{\max }=\sum_{i=1}^{n} \max _{\forall c s_{i, j} \in c s_{i}} q_{i j}^{k}
\end{aligned}
$$

Prior to the mutation operation, the utility of each concrete service in each service candidate set is computed. Then all the concrete services in each service candidate set are sorted in descending order according to their utility. When the mutation operation is applied, the replacement process will search another service candidate from the beginning of the service candidate set until the assignment is different from the old assignment, and then replace it.

The service selection algorithm based on a modified GA for data-intensive service composition is given in Fig. 6.

\section{PERformance EVAluation}

In order to evaluate the performance of the proposed algorithm, we verified it against the simulated composite service the functional graph of which is shown in Fig. 3. The loop structure can be unfolded by cloning the vertices involved in the structure as many times as the maximal loop count [14]. This paper does not deal with loop relation so the maximal loop count is 1 .

\section{A. Test case generation}

The values of parameters considered in this paper are: Npop $=20$, MaxIt $=1000, P C=0.7, P M=0.1$. The weights for QoS attribute cost and response time are 0.8 and 0.2 respectively. The performance of the modified genetic algorithm is affiliated to the size of the data-intensive service composition problem. The size of the problem depends on the number of abstract services in the workflow and the number of concrete services for each abstract service. Thus, we generate two test groups. The first test group includes 5 test scenarios with different numbers of service candidates. The number of service candidates for each abstract service ranges from 10 to 50 , in increments of 10 . The number of abstract services is fixed at 9 . The second test group includes 4 test scenarios with different numbers of abstract services. The number of abstract services is $15,20,25$ and 30 . This was done by increasing the number of abstract services on the workflow of Fig. 3 with a sequential flow structure. The number of service candidates for each abstract service is fixed at 10 . This two test groups are designed to test how the running time of the proposed

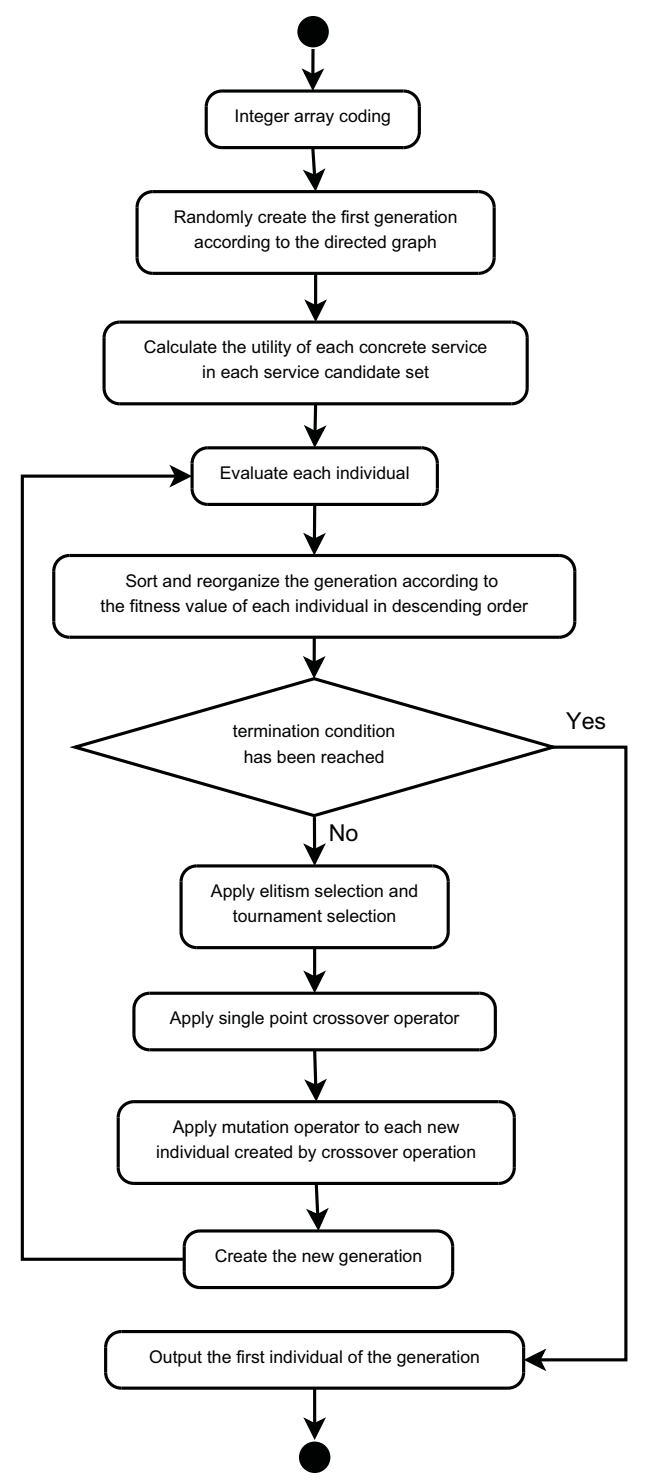

Fig. 6: Data-intensive service composition selection algorithm based on GA

algorithm will change as the number of service candidates and the number of abstract services change.

All test scenarios are run twenty times and the average values are reported. The cost and response time of a service candidate are randomly drawn from a uniform distribution of the interval $[1,10]$. The access cost and transfer time of each data set are randomly drawn from a uniform distribution of the interval $[1,10]$. The transfer cost is the price to be paid per unit of transfer time and its value is set as 1 in this paper. The values of the access cost and transfer time of data sets, and the values of the cost and response time of service candidates will not affect the running time or generations of the proposed algorithm. So their values can be in any intervals. 


\section{B. Result analysis}

The simulation results are shown in Fig. 7 on the next page. Fig. 7(a) to Fig. 7(e) show the results of the first test group. Fig. 7(f) to Fig. 7(i) show the results of the second test group. In Fig. 7(a) to Fig. 7(i), the blue line denotes the fitness value of the best individual from the beginning of the trial, and the red point denotes the fitness value of the best individual of each generation. The value of 'GUtility' is the fitness value of the best individual from the beginning of the trial and it depends on the QoS attributes of services. Different values of QoS attributes give different values of 'GUtility'. That is to say, the change of the value of 'GUtility' has no significance for the simulation results. The value of 'FRIT' is the number of generations when the best fitness value appeared and from this iteration the value of the best fitness will not change.

Table I gives the details of the results of the first test group. According to the value of 'Average FRIT' in Table I, as the number of concrete services increases from 10 to 50 in increments of 10 , the number of generations to create the best individual does not change, which is fixed at 7 . This is because, by using the integer array coding scheme, the change of the number of service candidates will not influence the length of the genome.

TABLE I: Number of service candidates, Fitness, Time, Generation

\begin{tabular}{|l|l|l|l|}
\hline $\begin{array}{l}\text { Number of service } \\
\text { Candidates }\end{array}$ & $\begin{array}{l}\text { Average } \\
\text { GUtility }\end{array}$ & $\begin{array}{l}\text { Average Run- } \\
\text { ning Time (s) }\end{array}$ & Average FRIT \\
\hline 10 & 0.91708 & 0.0112 & 7 \\
\hline 20 & 0.92750 & 0.0112 & 7 \\
\hline 30 & 0.96054 & 0.0112 & 7 \\
\hline 40 & 0.95072 & 0.0112 & 7 \\
\hline 50 & 0.96611 & 0.0112 & 7 \\
\hline
\end{tabular}

Table II gives the details of the results of the second test group. According to the value of 'Average FRIT' in Table II, as the number of abstract services increases, the number of generations to create the best individual also increases. Fig. 7(j) shows the effect of the number of abstract services on the running time for generating the best individual.

TABLE II: Number of tasks, Fitness, Time, Generation

\begin{tabular}{|l|l|l|l|}
\hline Number of tasks & $\begin{array}{l}\text { Average } \\
\text { GUtility }\end{array}$ & $\begin{array}{l}\text { Average Run- } \\
\text { ning Time (s) }\end{array}$ & Average FRIT \\
\hline 15 & 0.95852 & 0.0250 & 9 \\
\hline 20 & 0.96262 & 0.0335 & 10 \\
\hline 25 & 0.95235 & 0.0445 & 11 \\
\hline 30 & 0.93575 & 0.0582 & 12 \\
\hline
\end{tabular}

\section{CONCLUSION}

In this study, we continued our earlier work and presented a modified genetic algorithm along with the evaluation of its performance through simulations. We present the lifetime of data-intensive service composition and model the dataintensive service composition problem as a directed graph. We also present the optimization problem of data provider, service provider and service composer in the two-stage negotiation procedure. The proposed genetic algorithm adopts the combination of elitism selection and tournament selection, a modified crossover and mutation operations to find the optimal solution. Future extensions of the work will develop a local selection rule to improve the performance of the algorithm, and compare it with other approaches such as mixed integer programming and random selection approaches. Also, the algorithm will be applied in a real-life application. This paper tries to apply a modified GA to implement the first step in the whole process as discussed earlier, and the development of the algorithms for the two negotiation processes for data-intensive service composition are currently under way.

\section{ACKNOWLEDGMENT}

The authors wish to express their sincere gratitude to Dr. Madeleine Cincotta for her help in the editing of this manuscript.

\section{REFERENCES}

[1] R. F. Easley, and R. Tenorio, "Jump bidding strategies in internet auctions”, Management Science, Vol. 50, pp. 1407-1419, 2004.

[2] O. Gunther, G. Tamm, and F. Leymann, "Pricing web services", Int. J. Business Process Integration and Management, Vol. 2, No. 2, pp. 132-140, 2007.

[3] S. J. Kafka, B. D. Temkin, and L. Wegner, "Business-to-business auctions go beyond price", Forrester Research Inc., 2000.

[4] C. Preist, A. Byde, C. Bartolini, and G. Piccinelli, "Towards Agentbased Service Composition through Negotiation in Multiple Auctions", Technical Report HPL-2001-71, Hewlett Packard, 2001.

[5] C. Preist, C. Bartolini, and A. Byde, "Agent-based service composition through simultaneous negotiation in forward and reverse auctions", Proceedings of the 4th ACM conference on Electronic commerce, pp. 55-63, 2003.

[6] B. Prashanth, and Y. Narahari, "Efficient algorithms for combinatorial auctions with volume discounts arising in web service composition", Proceedings of the 4th IEEE conference on Automation Science and Engineering, pp. 995-1000, 2008.

[7] J. Shen, G. Beydoun, S. Yuan, and G. Low, "Comparison of bioinspired algorithms for peer selection in services composition", IEEE International Conference on Services Computing (SCC 2011), pp. 250-257, 2011.

[8] J. Shen, and S. Yuan, "QoS-aware peer services selection using ant colony optimisation", Business Information Systems Workshops, pp. 362-374. 2009.

[9] M. Srinivas, and L. M. Patnaik, "Genetic algorithms: a survey", IEEE computer, Vol. 27, pp. 17-26, 1994.

[10] L. Wang, J. Shen, and J. Yong, "A Survey on Bio-Inspired Algorithms for Web Service Composition", International Conference on Computer Supported Cooperative Work in Design, pp. 569-574, 2012.

[11] L. Wang, and J. Shen, "Towards Bio-Inspired Cost Minimisation for Data-intensive Service Provision", IEEE International Conference on Services Economics, pp.16-23, 2012.

[12] X. Wang, Z. Wang, and X. Xu, "Price heuristics for highly efficient profit optimization of service composition", IEEE International Conference on Services Computing, pp. 378-385, 2011.

[13] S. Yuan, J. Shen, and A. Krishna, "Ant inspired scalable peer selection in ontology-based service composition", IEEE World Congress on Services, pp. 95-102, 2009.

[14] L. Zeng, B. Benatallah, A. H. H. Ngu, M. Dumas, J. Kalagnanam, H. Chang, "QoS-aware middleware for Web services composition", IEEE Transactions on Software Engineering, Vol. 30, No. 5, pp. 311-327, 2004. 

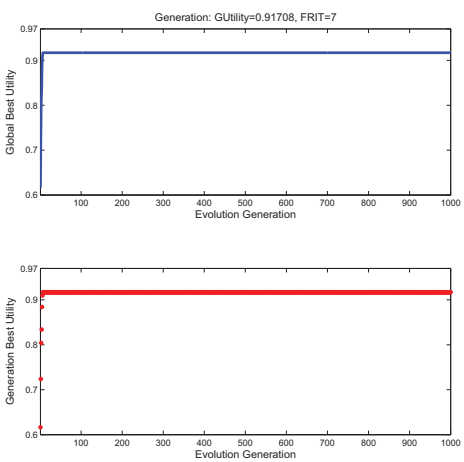

(a) 10 service candidates
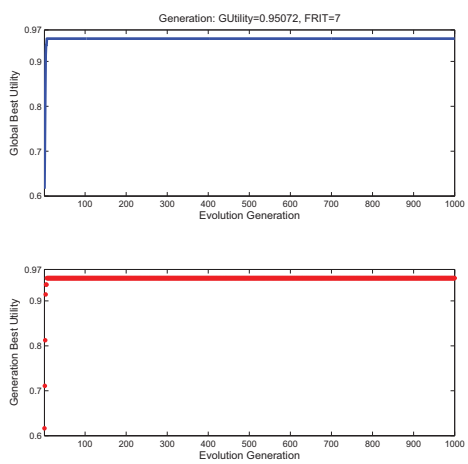

(d) 40 service candidates
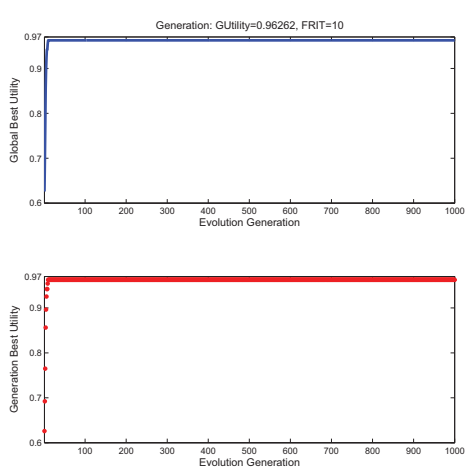

(g) 20 abstract services
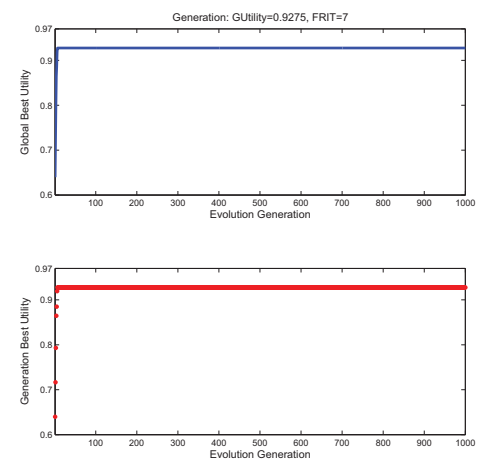

(b) 20 service candidates
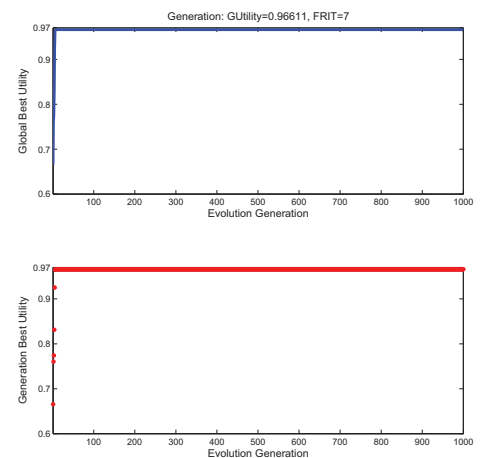

(e) 50 service candidates
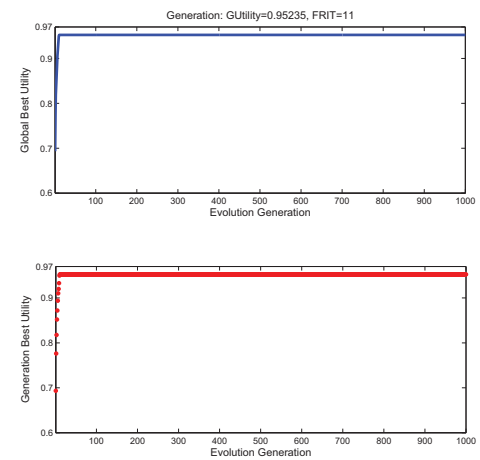

(h) 25 abstract services

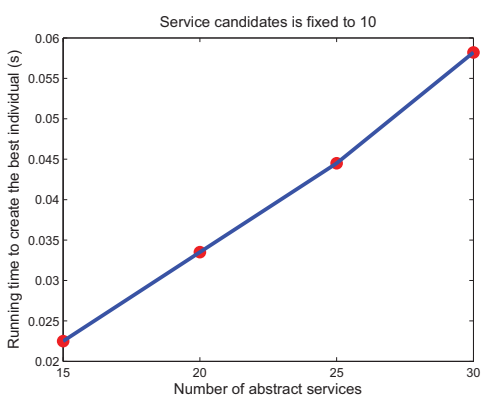

(j) The effect of the number of abstract services on the running time for generating the best individual.

Fig. 7: The results of the two test groups
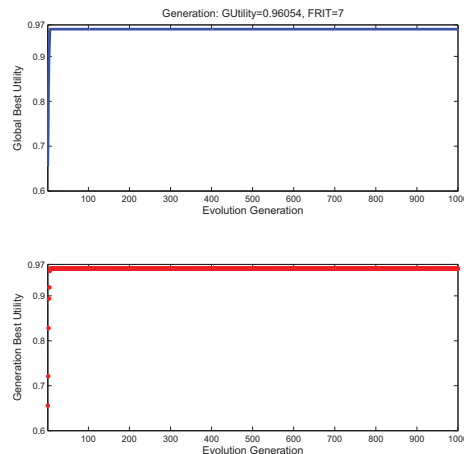

(c) 30 service candidates
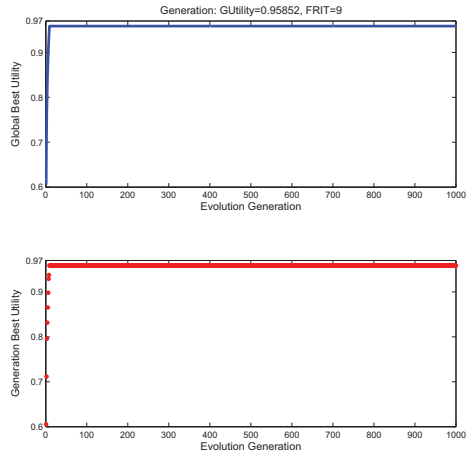

(f) 15 abstract services
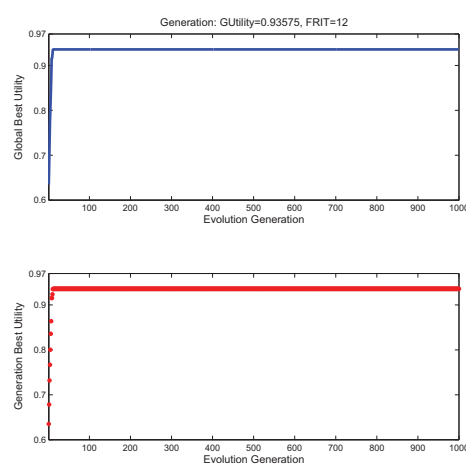

(i) 30 abstract services 